\title{
A Classification Proposal for Computer-Assisted Knee Systems
}

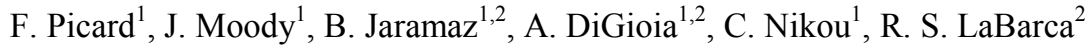 \\ Centers for Medical Robotics and Computer-Assisted Surgery, \\ ${ }^{1}$ UPMC Shadyside Hospital, Pittsburgh, PA and \\ ${ }^{2}$ Robotics Institute, Carnegie Mellon University, Pittsburgh, PA.
}

\begin{abstract}
Computer-Assisted Surgery (CAS) combines various enabling technologies to help surgeons meet and exceed quality requirements in performing knee surgery. Emerging CAS systems for the knee already include applications for TKR, ACL reconstruction and tibial osteotomies. In this paper we propose a general scheme to classify computer-assisted knee systems currently in use and under development, with an emphasis on surgical navigation systems. A literature review and available commercialized product analyses allowed us to sort the different computer-assisted systems in several categories. We proposed a classification scheme relies upon medical criteria instead of solely technical criteria (such as localizer properties and computer specifics). New concepts in computer-assisted surgery can easily fit into this classification framework.
\end{abstract}

Keywords: Computer-assisted knee surgery systems.

\section{Introduction}

Knee-related operations comprise a significant percentage of the routine orthopaedic surgeries performed daily in the United States ${ }^{1,2,3}$. Within this group two procedures predominate: Total Knee Replacement (TKR) for degenerative conditions, and Anterior Cruciate Ligament (ACL) reconstruction for ACL tears. Approximately 300,000 knee arthroplasties and 50,000 ACL reconstructions are performed every year in the $\mathrm{USA}^{4}$.

Making these knee procedures more accurate and less invasive would increase immediate and long-term success, thereby increasing the overall benefit to the patient. However, there remain several practical challenges to improving clinical techniques $^{5,6}$. Computer-assisted surgery has emerged as one of the key-solutions for solving these technical challenges. Over the last few years, several computer-assisted systems have been developed.

In this paper we propose a general scheme to classify computer-assisted knee systems currently in use and under development with an emphasis on surgical navigation systems.

\section{Background}

Computer-assisted technologies can be loosely classified in two categories: Robotic Assistive Systems and Surgical Navigation Systems. Sub-classifications within these 
groups can be determined by examining the underlying principles and functionality of various systems.

1. Robotic Assistive Systems:

Robotic systems are those in which an active manipulator is used in some aspect of the surgical procedure. Robotic systems come in direct contact with the patient and must necessarily employ patient-specific models. Several robotic systems have been developed for use in knee surgery to date.

1.1. TKR surgery:

Matsen $^{7}$ and coworkers were the first authors to describe a robotic system for knee arthroplasty using active robotic templates. Approximately at the same time Kienzle and Stulberg ${ }^{8}$ developed a robotic assisted system that utilized a preoperative CT scan and pin based registration technique. A 3-D reconstruction allowed them to define the ideal positioning and the ideal size of the prostheses. Other researchers (Davies ${ }^{9}$, Glozman $^{10}$ and Fadda/ Marcacci/ Martelli ${ }^{11}$ ) have used similar approaches. Afterwards, these teams used either an active robot or a semi-active ${ }^{9}$ robot in order to orient a drilling or milling tool to perform surgery according to a preoperative plan. The preliminary cadaver results showed accuracy to within $1 \mathrm{~mm}$ and out "degree". Following the same principles implemented by Paul and Bargar, Bauer et $\mathrm{al}^{29}$, Kober et al. ${ }^{30}$ described an active robot for milling bones.

\subsection{ACL Reconstruction Surgery:}

Two types of robotic techniques have been developed:

- An active robot for drilling the tibial and femoral tunnels has been developed by Peterman ${ }^{12}$ and coworkers. After obtaining a CT scan with pins previously applied, the authors used a calibrated robot to drill bones in the ideal position for anchoring the ACL graft. The first clinical trial involved 25 patients. Gotte and al. $^{13}$ following the same principles have developed a similar robotic technique.

- Active robot for leg stabilization: using a robotic manipulator, Sakane ${ }^{14}$ and al. proved the reliability of robotic technique used to provide ideal knee positioning during the ACL reconstruction on cadavers. The goal was to stabilize the knee in a good position during the surgical procedure in order to improve the location of the graft.

2. Surgical Navigation Systems

Surgical Navigation Systems essentially provide intraoperative guidance. These systems typically use optical or magnetic markers to track tools and patient anatomy. Surgical plan may be preoperatively generated, intraoperatively generated, or both (preoperative data refined intraoperatively). Several knee surgical navigation systems currently exist.

2. 1. Surgical Navigation Systems based on Preoperative Models:

2.1.1. Total Knee Arthroplasty:

$\mathrm{KneeNav}^{\mathrm{TM}}$, an image guided navigation system using optoelectronic tracking, for TKR (and also ACL reconstruction) under development in the Centers for Medical Robotics and Computer Assisted Surgery at Carnegie Mellon University and UPMC Shadyside Hospital. KneeNav relies on the same principles as the HipNav ${ }^{15}$ system, a system successfully used for total hip replacement surgery. 
A similar guided navigation system named Navitrack $^{\mathrm{TM}}{ }^{23}$ for $\mathrm{TKR}$ has been developed by Orthosoft, Inc, Montreal, Canada. This system relies upon an imageguided navigation system using electromagnetic tracking.

Other researchers have worked on applications like high tibial osteotomies, (Tso and Ellis ${ }^{18}$ ) using $\mathrm{CT}$ images to perform computer-assisted surgical navigation.

2.1.2. ACL reconstruction:

Sati $^{16}$ et al. proposed a system that utilizes preoperative planning based on CT Scan information. The CT scan is used for registration and 3D visualization of the patient anatomy. Using a magnetic tracking system, the computer was able to follow knee movement in real time. A graphical interface displayed the 3D image of the knee and allowed the analysis of the knee motion in real time. The system also predicts elongation properties and isometric points of the ligaments. This system is currently not in clinical use.

2.2. Surgical Guided Systems based on Intraoperative Model:

2.2.1. Total Knee Arthroplasty:

Picard and Leitner ${ }^{19,24,25,31}$ described the first clinically used computer-assisted system for performing a complete TKR procedure. No preoperative imagery was required. The principle was to determine the mechanical axes of the limb during the surgical procedure and then to orient pre-calibrated cutting guides in reference to those axes using optoelectronic guidance. Real-time user interfaces presented the mechanical axes and the orientation of the cutting guides. The surgeon then secured the jigs using the computer guidance system and extra-medullary instrumentation. The bone was cut using traditional oscillating saws.

- Krackow ${ }^{20}$ and coworkers developed a similar system, also without the use of preoperative imagery. An optoelectronic localizer was used to track bones and tools during the surgical procedure. Two rigid bodies (tracking markers) are fixed to the distal femur and the proximal tibia. The center of the femoral head was found by moving the femur around the hip joint. The author utilized a traditional cannulated intramedullary femoral rod oriented with computer guidance to perform the surgery.

- Kuntz, Sati, and Nolte ${ }^{28}$ developed a similar system. For registration of the hip center they used a pivoting algorithm that does not require a reference base on the pelvis.

\subsubsection{ACL reconstruction:}

Dessenne and Julliard ${ }^{21}$ used an optical tracking system to determine the ideal placement of the ACL during the usual surgical procedure, with the help of an isometry map. Using a pre-calibrated probe, the surgeon recorded anatomical data (the points on the knee surfaces) during the arthroscopic procedure, and then by moving the knee computed the relative position between femur and tibia so that define an isometry map. System was also able to calculate the position of the graft relative to the tibial and femoral holes (tension of the graft) and to the roof (impingement).

Klos and Banks ${ }^{17}$ developed a system based on fluoroscopic images. During the surgical procedure they were able to define ideal placement and isometry of the ACL graft, using an overlap template, which coincides with the lateral fluoroscopic view. The system tracks the surgical instruments within the fluoroscopic image, and 
generates graphic overlays. The results of the clinical trial showed an accuracy of $1.1 \mathrm{~mm}$.

Fleute $^{22}$ et al: Using ten cadaver femurs authors generated a generic shape of the femur. Intraoperatively, point collections and a registration process of the distal femur allowed them to match the ideal fitting between intraoperative registration and generic shape. A statistical model was used to ideally match the femoral registration and the generic shape.

\section{Results}

Based on our survey of existing and developing computer-assisted technologies, we propose the classification according to the following categories ${ }^{27,32}$ :

1- Robotic Assistive Systems are robotic devices that perform a surgical task according to some preoperative data. Three classifications have been defined in the literature:

1.1. Active robotic system perform some surgical task, such as drilling or milling, without the direct intervention of the surgeon.

1.2. Semi-active robotic systems augment the surgeon's control of the tool. Such a system may, for example, not directly control a saw during a resection, but may limit the depth of cut. Most systems in this category restrict a task within a pre-determined envelope. In other words they enforce constraints.

1.3. Passive robotic system performs some part of the surgical procedure while under continuous and direct control of the surgeon but does so under the direct guidance or control of the surgeon. It may, for example be used to position a template or cutting guide block.

- Surgical Navigation Systems are computer-assisted systems that display information for orientation and guidance during the surgical procedure. They may present accurate anatomical images, simple graphics, or a combination of images (for example, icons superimposed on radiographic data). These can be loosely classified into two groups depending on their use of models:

2.1. Preoperative-model systems rely on models generated preoperatively, usually from large data sets (CT or MRI image series, for example). They can provide a wealth of detailed information, and are typically used in conjunction with a planning system. Preoperative model system can be either patient-specific or not patientspecific.

2.1.1. Patient-specific means that the preoperative model is set up from specific data of the patient itself. For example, the preoperative plan can be based on CT images of the patient and this data will be used as a reference during the surgical procedure.

2.1.2. Non patient-specific means that the preoperative model is based on a generic shape or generic model. A homothetic model resulting from a generic model derived from images or digitized cadaver bones can be used during the surgical procedure.

2.2. Intraoperative-model systems develop anatomical models through intraoperative data collection. Intraoperative model systems can be divided in two categories depending on their use of intraoperative images. 
2.2.1. Image based: an intraoperative image (such as a set of coordinated fluoroscopic images ${ }^{26}$ ) are generated during the surgical procedure and used as a frame of reference.

2.2.2. Non-image based: all model information required for the task is determined from direct measurement of the bone surface (using, for instance a calibrated and tracked probe), or from direct measurement of limb dynamics (e.g., computing rotational centers from relative bone movement).

\section{Discussion}

Table I summarizes the classification breakdown. This classification scheme relies upon medical criteria instead of solely technical criteria (such as localizer properties and computer specifics).

Table I: Classification of Computer-Assisted Knee Systems.

\begin{tabular}{|c|c|c|c|c|}
\hline $\begin{array}{c}\text { Robotic Assistive } \\
\text { Systems }\end{array}$ & \multicolumn{3}{|c|}{ Surgical Navigation Systems } \\
\hline Passive Systems & \multicolumn{2}{|c|}{$\begin{array}{c}\text { Preoperative-model } \\
\text { Systems }\end{array}$} & \multicolumn{2}{|c|}{ Intraoperative-model Systems } \\
\cline { 1 - 3 } Semi-active Systems & $\begin{array}{c}\text { Patient } \\
\text { Specific }\end{array}$ & $\begin{array}{c}\text { Non } \\
\text { patient } \\
\text { specific }\end{array}$ & $\begin{array}{c}\text { Intraoperative } \\
\text { Images }\end{array}$ & $\begin{array}{c}\text { No } \\
\text { Intraoperative } \\
\text { Images }\end{array}$ \\
\cline { 1 - 2 } Active Systems & & & \\
\hline
\end{tabular}

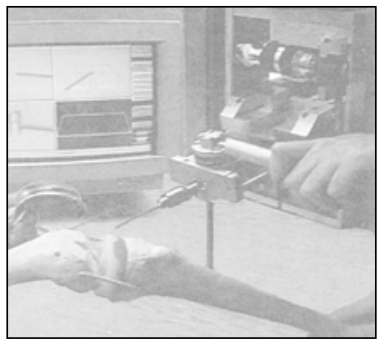

Fig. 1: Robotic Assistive System (Guided tools).

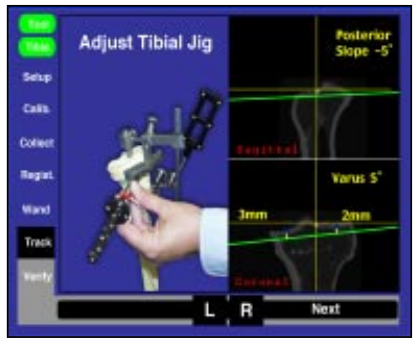

Fig. 2: Preoperative model Image guided model.

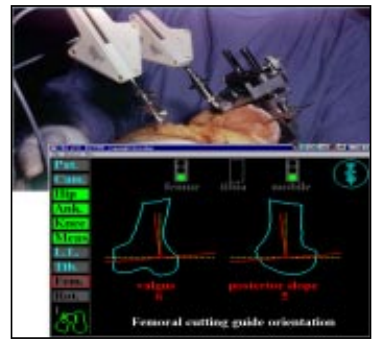

Fig. 3: Intraoperative model None Image guided based.

\section{Conclusion}

We propose a classification scheme for computer assisted systems for knee reconstructive surgery, based on a survey of systems currently in use or in development. The approach provides easy comprehension of technological requirements and the medical utility for a particular computer-assisted system. New concepts in computer-assisted surgery can easily fit into this classification framework. 


\section{References}

1. Ayers D.C., Berman A.T., Duncan C.P., and al., Economic Aspects of Total Joint Replacement. AAOS Committee on Hip and Knee Arthritis, 1997.

2. Lavernia C.J., Guzman J.F., Gachupin-Garcia A., Cost Effectiveness and Quality of Life in Knee Arthroplasty. Clin. Orthop. Relat. Res., 345, 134-139, 1997.

3. Rorabeck C.H., Murray P., The Cost Benefit of Total Knee Arthroplasty. Orthopedics, 19 (9), 777-779, 1996.

4. Frank C.B, Jackson D.W., The Science of Reconstruction of the Anterior Cruciate Ligament. J. Bone J. Surg, 79-A (10), 1556-1576, 1997.

5. Ayers D.C, Dennis D.A., Johanson N.A and al., Common Complications of Total Knee Arthroplasty. J. Bone J. Surg., 79-A (2), 278-311, 1997.

6. Andersson C., Odensten M., Gillquist J., Knee function after surgical or non-surgical treatment of acute rupture of the ACL: A randomized study with a long-term follow-up period. Clin. Orthop., 264, 1991.

7. Matsen F.A., Garbini J.L., Sidles J.A, and al. Robotic Assistance in Orthopaedic Surgery. (A proof of principle using distal femoral arthroplasty). Clin. Orthop. Relat. Res., 296, 178-186, 1993.

8. Kienzle T.C., Stulberg S.D., Peshkin M. and al., A Computer-Assisted Total Knee Replacement Surgical System Using a Calibrated Robot. Orthopaedics. In computer Integrated Surgery, Cambridge, Massachusetts, The MIT Press. R.H. Taylor and al. Editor. 409-416, 1996.

9. Davies B.L., Harriss J., Lin W.J., and al., Active compliance in Robotic Surgery- The use of force control as a dynamic constraint. Journal of Engineering in Medicine, Proceedings H. of the Institution of Mechanical Engineers, UK, Volume 211, page H 4, November 1997.

10. Glozman D., Shoham M., Fischer A., Efficient registration of 3-D objects in RoboticAssisted Surgery. Proceedings CAOS 'US, Editor UPMC Shadyside Medical Center (A.DiGioia) 248-252, 1999

11. Fadda M., Bertelli D., Martelli S., Marcacci .M., and al., Computer Assisted Planning for Total Knee Arthroplasty. In First Joint Conference of CVRMed and MRCAS, Grenoble, France: Springer, 619-628, 1997.

12. Peterman J., Kober R., Heinze and al., Computer Assisted Planning and Robot Assisted Surgery in the Reconstruction of the Anterior Cruciate Ligament. Operative Techniques in Orthopaedics, 50-55, Vol 10, No 1(January), 2000.

13. Gotte H., Roth M., Brack C.H., and al. A new less-invasive approach to knee surgery using a vision-guided manipulator. IARP workshop on Medical Robotics, Vienna (Austria) Oct. 99-106, 1996.

14. Sakane M., Li G., Fox R., Woo L-Y., and al., The advantage of Robot - Assisted Knee Positioning for ACL Reconstruction Surgery. Advances in Bioengineering. ASME (33), 397-398, 1996.

15. DiGioia A., Jaramaz B., Blackwell M., and al., Image Guided Navigation System to Measure Intraoperatively Acetabular Implant Alignment. Clin. Orthop. and Relat. Res., 355, 8-22, 1998.

16. Sati M., DeGuise J.A., Drouin G., Computer Assisted Knee Surgery. Computer Aided Surgery 2: 108-123, 1997.

17. Klos T.V.S., Banks A.Z., Banks S.A., and al., Computer and Radiographic Assisted Anterior Cruciate Ligament Reconstruction of the Knee. In Computer Assisted Orthopaedic Surgery L.P. Nolte and R.Ganz Hogrefe\& Huber Publishers.184-189, 1999.

18. Tso C.Y., Ellis R.E., Rudan J., and al., A Surgical Planning and Guidance System for High Tibial Osteotomies. In Proceedings Medical Image Computing and Computer-Assisted Intervention- MICCAI'98, 39-50, 1998. 
19. Leitner F, Picard F., Minfelde R. and al. Computer-Assisted Knee Surgical Total Replacement. In First Joint Conference of CVRMed and MRCAS, Grenoble, France: Springer, 629-638, 1997.

20. Krackow K., Serpe L., Phillips M.J., and al., A New Technique for Determining Proper Mechanical Axis Alignment During Total Knee Arthroplasty. Orthopedics, 22 (7), 698$701,1999$.

21. Dessenne V., Lavallee S., Julliard R., and al., Computer Assisted Knee Anterior Cruciate Ligament Reconstruction: First Clinical Tests. Journal of Image Guided Surgery 1, 59-64, 1995.

22. Fleute M., Lavallee S., Julliard R., Incorporating a statiscally-based shape model into a system for computer-assisted anterior cruciate ligament surgery. Medical Image Analysis, Oxford University Press volume 3, 209-222, 1999.

23. Amiot L.P., Labelle H., DeGuise J.A., Sati M., and al., Computer-assisted Pedicle Screw Fixation: a feasibility Study, Spine, 10, 1208-1212, 1995.

24. Stulberg S.D, Picard F., Saragaglia D. Computer-Assisted Total Knee Arthroplasty. Operative Techniques in Orthopaedics, 25-39, Vol 10, No 1(January), 2000.

25. Delp S.L., Stulberg S.D., Davies B., Picard F., Leitner F., Computer Assisted Knee Replacement. Clin. Orthop. Relat. Res., 354, 49-56, 1998.

26. Hofstetter R., Slomczykowski M., Sati M., Nolte L.P., Fluoroscopy as an Imaging Means for Computer-Assisted Surgical Navigation. Computer Aided Surgery., 4:65-76, 1999.

27. Troccaz J. Man-Machine Interfaces in Computer-Augmented Surgery. In Computer Assisted Orthopaedic Surgery L.P. Nolte and R.Ganz Hogrefe \& Huber Publishers. 53-68, 1999.

28. Kuntz M., Sati M., Nolte L-P., and al. Computer Assisted Total Knee Arthroplasty. In International Symposium on CAOS, Davos, February, 17-19, 2000.

29. Bauer A., Robot-Assisted Total Hip Replacement in Primary and Revision Cases. Techniques in Orthopaedics, 9-13, Vol 10, No 1(January), 2000.

30. Kober R., Meister D., Total Knee Replacement using the Caspar-system. Computer Assisted Total Knee Arthroplasty. In International Symposium on CAOS, Davos, February 17-19, 2000.

31. Picard F., Leitner F., Raoult O., Saragaglia D. Computer-Assisted Knee Replacement. Location of a rotational center of the knee. Total Knee Arthroplasty. In International Symposium on CAOS, Davos, February 17-19, 2000.

32. Taylor R.H., Robotics in Orthopedic Surgery, In Computer Assisted Orthopaedic Surgery (CAOS), L.P. Nolte and R.Ganz, 35-41, 1998. 